\title{
SIZE DEPENDENCE OF THE ENERGETICS OF ELECTRON ATTACHMENT TO LARGE WATER CLUSTERS
}

\author{
R.N. BARNETT, Uzi LANDMAN, C.L. CLEVELAND \\ School of Physics, Georgia Institute of Technology, Atlanta, GA 30332, USA
}

and

Joshua JORTNER

School of Chemistry, Sackler Faculty of Exact Sciences, Tel Aviv University, 69978 Tel Aviv, Israel

Received 20 January 1988

\begin{abstract}
The size dependence of the binding energy of a localized excess electron in large water clusters originates from long-range polarization interactions. The vertical and adiabatic binding energies of compact, interior excess electron states in $\left(\mathrm{H}_{2} \mathrm{O}\right)_{n}^{-}$clusters, obtained from quantum path-integral molecular-dynamics simulations, exhibit a linear dependence on $n^{-1 / 3}$, in quantitative agreement with the implications of dielectric medium effects in finite systems.
\end{abstract}

The existence of the solvated electron was experimentally demonstrated in 1863 for liquid ammonia [1] and in 1962 for water [2]. Electron localization in these and other polar fluids [3-7] is non-reactive, involving the combination of long-range [8] and short-range [9-12] attractive interactions, and is accompanied by a large solvent reorganization $[9,10]$. During the last decade the problem of electron localization in a finite clusters of polar molecules, e.g. $\left(\mathrm{H}_{2} \mathrm{O}\right)_{n}$ or $\left(\mathrm{NH}_{3}\right)_{n}$ [13-17], constituted an experimental and theoretical challenge for two reasons. Firstly, such large finite systems provide ways and means for the elucidation of microscopic solvation effects of an excess electron in a well defined, finite solvent environment. Secondly, the phenomenon of electron attachment to molecular clusters falls in the category of size effects on chemical and physical phenomena, pertaining to the issue of the minimal size of a (water or ammonia) cluster which can sustain a bound state of an excess electron.

We have recently applied the quantum path-integral molecular-dynamics (QUPID) method [18-28] to explore the localization modes of an excess electron in $\left(\mathrm{H}_{2} \mathrm{O}\right)_{n}$ clusters over a broad range of cluster sizes $(n=8-128)$ and over a wide temperature do- main $(T=79-300 \mathrm{~K})$ [26-28]. A major conclusion which emerged from our previous studies [26-28] was that electron localization in medium-sized $(8 \leqslant n \leqslant 32)$ water clusters constitutes a novel excess electron surface state on the water clusters and only on increasing the size of the water clusters above $n>32$ does the interior electron localization mode become energetically favored. The interior electron localization mode in large clusters constitutes the precursor of the celebrated solvated electron [3-12]. The size dependence of the binding energies of interior electron states in water clusters, which is the subject matter of this note, provides information regarding the role of long-range attractive "large-polaron" interactions [8] on electron solvation in clusters of polar molecules and in polar fluids.

The energetics and structure of excess electron states in $\left(\mathrm{H}_{2} \mathrm{O}\right)_{n}^{-}$clusters were obtained from the QUPID simulations. The QUPID method rests on an isomorphism between the quantum problem and a classical one, wherein the quantum particle is represented by a necklace of $P$ pseudo-particles ("beads") with nearest-neighbor harmonic interactions [18-26]. Invoking previous formalism and no- 
tation $[21,26-28]$, the average total energy of the system is

$$
E=3 N / 2 \beta+\left\langle V_{\mathrm{c}}\right\rangle+K+P^{-1} \sum_{i=1}^{P}\left\langle V\left(r_{i}\right)\right\rangle
$$

with $K=3 / 2 \beta+K_{\text {int }}$, where $V_{\mathrm{c}}$ is the interaction potential between the classical particles (whose number is $n), V\left(\boldsymbol{r}_{i}\right)$ is the cluster-electron interaction for the $i$ th pseudoparticle,

$K_{\mathrm{int}}=\frac{1}{2 P} \sum_{i=1}^{P}\left\langle\left(\partial V\left(r_{i}\right) / \partial r_{i}\right) \cdot\left(r_{i}-r_{P}\right)\right\rangle$,

$\beta=1 / k T$ and $\langle>$ indicates statistical averaging. The water molecules in this study were treated classically. The choice of the number, $P$, of beads representing the excess electron is temperature dependent. As a rule of thumb, we found that for the clectron-water system an adequate discretization of the electron path is achieved for $P k T \geqslant e^{2} / a_{0}$. A key factor in modeling the system is the choice of interaction potentials. We have used the RWK2-M model $[29,30]$ for the intra- and inter-molecular interactions. For the electron-water interaction we have constructed a local pseudo-potential in the spirit of density functional theory, which consists of Coulomb, polarization, exclusion, and exchange contributions [26-28]. The details of the interaction potentials and the QUPID calculations for $\left(\mathrm{H}_{2} \mathrm{O}\right)_{n}^{-}$clusters have been described elsewhere [26-28].

The energetics of the $\left(\mathrm{H}_{2} \mathrm{O}\right)_{n}^{-}$clusters is expressed in terms of the electron vertical binding energy (EVBE), electron adiabatic binding energy $(\mathrm{EABE})$ and cluster reorganization energy $\left(E_{\mathrm{c}}\right)$,

$\mathrm{EVBE}=K_{\mathrm{int}}+\langle V\rangle$,

$\mathrm{EABE}=\mathrm{EVBE}+E_{\mathrm{c}}$,

$E_{\mathrm{c}}=\left\langle V_{\mathrm{c}}\right\rangle_{\left(\mathrm{H}_{2} \mathrm{O}\right)_{n}^{-}}-\left\langle V_{\mathrm{c}}\right\rangle_{\left(\mathrm{H}_{2} \mathrm{O}\right)_{n}}$.

The cluster reorganization energy (eq. (3)) is the difference between the equilibrium intramolecular and intermolecular potential energies in the negatively charged $\left(\left(\mathrm{H}_{2} \mathrm{O}\right)_{n}^{-}\right)$and the corresponding equilibrium neutral $\left(\left(\mathrm{H}_{2} \mathrm{O}\right)_{n}\right)$ clusters. - EVBE is the energy required to detach the electron from the $\left(\mathrm{H}_{2} \mathrm{O}\right)_{n}$ clusters without allowing nuclear rearrangement to occur and is the quantity measured in pho- toelectron spectroscopy [15] and EABE is the heat of solution of the excess electron in the cluster. The energetic stability of the negatively charged cluster with respect to the equilibrium neutral cluster plus free electron is inferred from the magnitude and sign of $\mathrm{EABE}$, with negative value corresponding to an energetically stable bound state.

The size dependence of the EABE interior $\left(\mathrm{H}_{2} \mathrm{O}\right)_{n}^{-}$states is summarized in fig. 1 , where we have also presented the energetic data for the surface excess electron states. From these data we conclude that (1) interior $\left(\mathrm{H}_{2} \mathrm{O}\right)_{n}^{-}$clusters are energetically unstable, i.e. EABE $>0$ for $n<20$. (2) For mediumsized clusters $8 \leqslant n \leqslant 32$ the electron localization mode involves the formation of a surface state, which is energetically stable [26-28]. (3) For large $n>64$ clusters the interior excess electron state is energetically favored. (4) The "transition" from energetically favored surface states to energetically stable interior states is exhibited in the range of cluster sizes $32<n<64$ where EABE is practically indentical for these two localization modes. A pictorial representation of the configuration of interior excess electron states in large water clusters $(n=64,128)$ is pre-

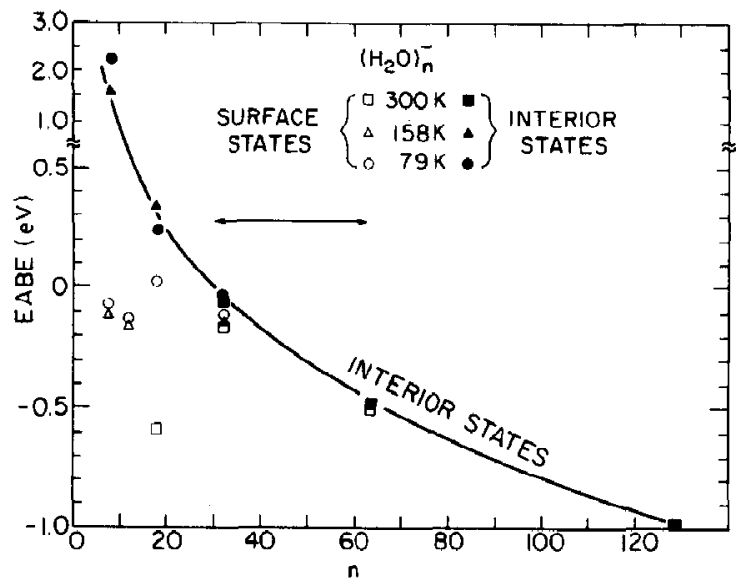

Fig. 1. The size dependence of the adiabatic binding energies of excess electrons in equilibrium $\left(\mathrm{H}_{2} \mathrm{O}\right)_{\vec{n}}(n=8-128)$ clusters. Open symbols refer to surface states while black symbols refer to interior states, as labelled on the figure. (The results for the interior state of the $\left(\mathrm{H}_{2} \mathrm{O}\right)_{8}^{-}$were obtained for a static configuration of molecules as used in ref. [31].) Energetically stable surface states are exhibited for medium-sized clusters $(8 \leqslant n \leqslant 30)$, for $n=32$ and 64 the energies of surface and interior states are about equal, while for large $(n=128)$ clusters the interior state is energetically stable. The region of crossover from surface to interior states is marked by a horizontal double arrow. 
sented in fig. 2. These interior states demonstrate a high degree of excess electron localization. Furthermore, it is interesting to note the development of hydrogen-bonded rings, which are characteristic of ice structure.

The dependence of the EABEs of interior electron states in clusters (fig. 1 ) reveals a monotonic increase of $|\mathrm{EABE}|$ with increasing $n$. We note that even for the largest $\left(\mathrm{H}_{2} \mathrm{O}\right)_{128}$ clusters studied by us the value of $\mathrm{EABE}=-0.98 \mathrm{eV}$ is considerably higher than the experimental value of the heat of solution $\Delta H=-1.70 \mathrm{eV}$ of the electron in bulk water [32]. These features qualitatively demonstrate the role of long-range polarization interactions in the energetic stabilization of the excess electron in large clusters and in the bulk.

The contribution of long-range polarization interactions to the binding energy of an electron in a large water cluster can be described in terms of the energy of an excess electron in a dielectric sphere. Electron attachment to surface [33,34] and interior [35] states in dielectric spheres was addressed. We consider electron localization in an interior state in a water cluster whose (average) cluster radius $\bar{R}$ considerably exceeds the spatial extent of the electron charge distribution, which can be characterized in terms of the radius of gyration of the electron necklace $R_{\mathrm{g}}$, i.e. $\bar{R} \gg R_{\mathrm{g}}$. In what follows we shall apply the theory of dielectric medium effects [36] to the large finite cluster. Assuming spherical symmetry of the electron charge distribution and uniform dielectric properties of the cluster, the adiabatic binding energy of the excess electron can be obtained from the total energy of an electron in a dielectric sphere

$\operatorname{EABE}(\bar{R})=K_{\mathrm{int}}-\frac{1}{2}\left(1-D_{\mathrm{s}}^{-1}\right) \int_{0}^{R} \mathrm{~d} r r^{2} E_{\mathrm{e}}^{2}(r)$,

where $K_{\text {int }}=K-3 / 2 \beta, D_{\mathrm{s}}$ is the static dielectric constant and $E_{0}(r)$ is the magnitude of the electric field due to the excess electron charge distribution. The vertical reorganization energy is related to EABE by eq. (2). The medium reorganization energy within the framework of the dielectric continuum theory is given in terms of the contribution of the orientational component of the polarization field [8]. $E_{\mathrm{c}}$ corresponds to the energy change involved in the transformation of a neutral dielectric sphere, in which both electronic and molecular orientation components of the polarization field are in equilibrium, to a non-equilibrium state of the neutral dielectric sphere, in which the electronic component of the polarization is in equilibrium while the orientational component of the polarization field attains its value in the equilibrium negatively charged system. Dielectric solvation theory gives [36]
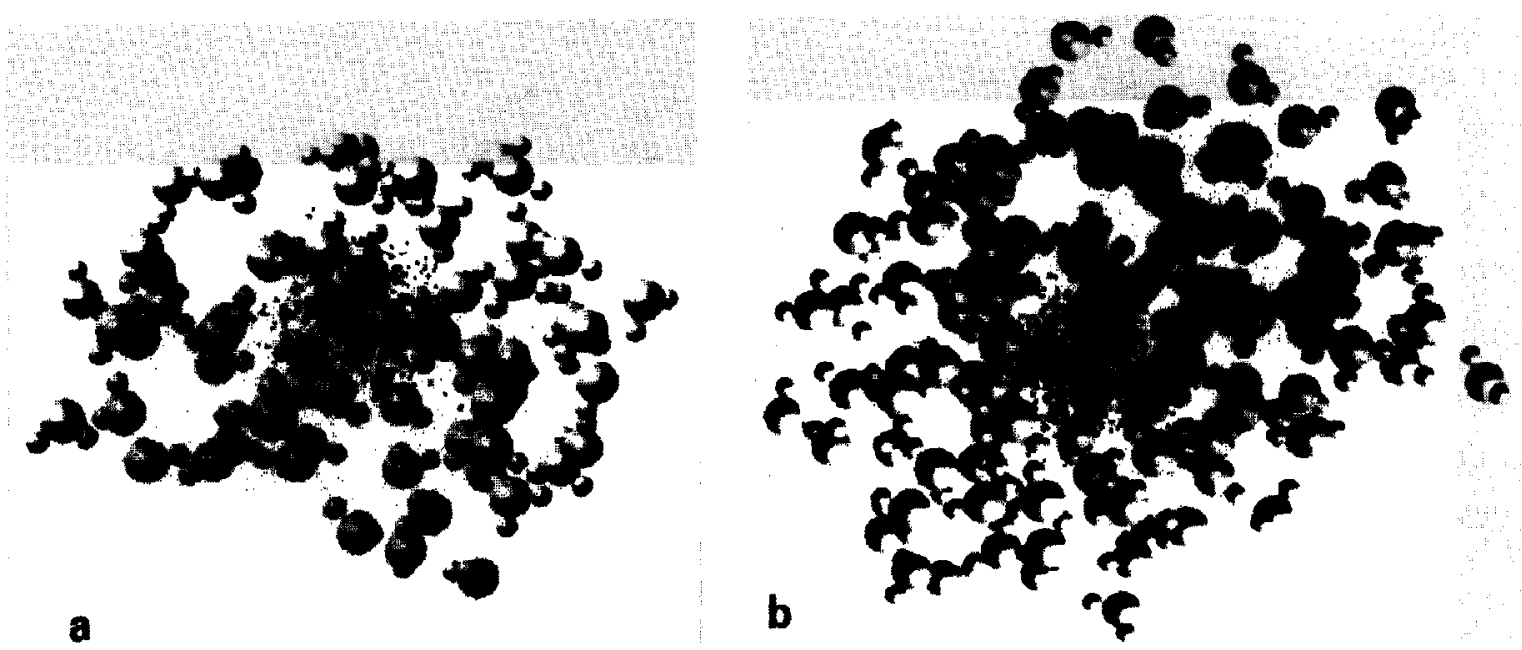

Fig. 2. Cluster configuration of $\left(\mathrm{H}_{2} \mathrm{O}\right)_{n}^{-}$obtained via quantum path-integral molecular-dynamics simulations. Large and small balls correspond to oxygen and hydrogen atoms, respectively. The dots represent the electron (bead) distributions. (a) Interior state in $\left(\mathrm{H}_{2} \mathrm{O}\right)_{\overline{64}} \cdot$ (b) Interior state in $\left(\mathrm{H}_{2} \mathrm{O}\right)_{\overline{128}}$. 
$E_{\mathrm{c}}(\bar{R})=\frac{1}{2}\left(D_{\mathrm{op}}^{-1}-D_{\mathrm{s}}^{-1}\right) \int_{0}^{\bar{R}} \mathrm{~d} r R^{2} E_{\mathrm{e}}^{2}(r)$,

where $D_{\mathrm{op}}$ is the optical dielectric constant. Eqs. (2), (4) and (5) result in

\section{$\operatorname{EVBE}(\bar{R})=\operatorname{EABE}(\bar{R})$}

$$
-\frac{1}{2}\left(D_{\mathrm{op}}^{-1}-D_{\mathrm{s}}^{-1}\right) \int_{0}^{R} \mathrm{~d} r r^{2} E_{\mathrm{e}}^{2}(r) .
$$

The relevant energies can be expressed in terms of the vertical and adiabatic binding energies for the infinite bulk system, which will be denoted by $\operatorname{EVBE}(\infty)$ and $\operatorname{EABE}(\infty)$, respectively, and which are given by eqs. (4) and (6) with $\vec{R}=\infty$. For sufficiently large clusters we observed [28] that the kinetic energy of the excess electron is independent of $\bar{R}$. This is due to the dominant role of the short-range interactions between the electron and the innermost shells of water molecules in localizing the electron. The spatial variation of the potential due to the longrange contribution, over the localized excess electron distribution, is exceedingly small. Accordingly, we get

$$
\begin{aligned}
& \operatorname{EABE}(\bar{R})=\operatorname{EABE}(\infty) \\
& +\frac{1}{2}\left(1-D_{\mathrm{s}}^{-1}\right) \int_{R}^{\infty} \mathrm{d} r r^{2} E_{\mathrm{e}}^{2}(r),
\end{aligned}
$$

$$
\operatorname{EVBE}(\bar{R})=\operatorname{EVBE}(\infty)
$$

$$
+\frac{1}{2}\left(1+D_{\mathrm{op}}^{-1}-2 D_{\mathrm{s}}^{-1}\right) \int_{R}^{\infty} \mathrm{d} r r^{2} E_{\mathrm{e}}^{2}(r) .
$$

In order to establish the connection between the energetics of electron attachment to a dielectric sphere, eqs. (7) and (8), and the properties of an excess electron in a cluster, we shall focus on a compact, localized, interior electron charge distribution in a large cluster (fig. 2), for which

$\rho(r)=0$ for $r>R_{1}$ and $R_{1}<\bar{R}$.

Condition (9) implies that the size effects on the energetics, which are expressed in terms of the integrals in eqs. (7) and (8), constitute an appropriate description of the physical situation. Accordingly, the energetics of electron attachment to a large water cluster can be adequately expressed in terms of eqs. (7) and (8) where $\operatorname{EVBE}(\infty)$ and $\operatorname{EABE}(\infty)$ are identified with the photoelectric threshold and the electron heat of solution in bulk water, respectively. Both observables can be inferred from QUPID calculations with realistic potentials or from experimental data.

To complete our analysis we shall provide explicit expressions for the electric field in the range $r>R_{1}$. Condition (9) implies that in this range $E_{\mathrm{e}}(r)=$ $r^{-2} \int \mathrm{d}^{3} r^{\prime} \rho\left(r^{\prime}\right)=e / r^{2}$. Eqs. (7) and (8) now result in

$$
\begin{aligned}
& \operatorname{EABE}(\bar{R})=\operatorname{EABE}(\infty)+\left(e^{2} / 2 \bar{R}\right)\left(1-D_{\mathrm{s}}^{-1}\right), \\
& \operatorname{EVBE}(\bar{R})=\operatorname{EVBE}(\infty) \\
& \quad+\left(e^{2} / 2 \bar{R}\right)\left(1+D_{\mathrm{op}}^{-1}-2 D_{\mathrm{s}}^{-1}\right) .
\end{aligned}
$$

Alternative useful expressions relate the energetics to the number, $n$, of polar molecules in the cluster. Setting $\bar{R}=r_{\mathrm{s}} n^{1 / 3}$, where $r_{\mathrm{s}}$ is the (mean) radius of the water molecule, results in linear relations of the energies versus $n^{-1 / 3}$,

$$
\begin{aligned}
& \operatorname{EVBE}(\bar{R})=\operatorname{EVBE}(\infty)+A n^{-1 / 3}, \\
& \operatorname{EABE}(\bar{R})=\operatorname{EABE}(\infty)+B n^{-1 / 3},
\end{aligned}
$$

where

$$
\begin{aligned}
& B=\left(e^{2} / 2 r_{\mathrm{s}}\right)\left(1-D_{\mathrm{s}}^{-1}\right), \\
& A=\left(e^{2} / 2 r_{\mathrm{s}}\right)\left(1+D_{\mathrm{op}}^{-1}-2 D_{\mathrm{s}}^{-1}\right) .
\end{aligned}
$$

An estimate of $r_{\mathrm{s}}$ can be obtained from the average density of molecules in the interior of a large water cluster yielding [28] $r_{\mathrm{s}} \approx 23.5 a_{0}$, while Newton recommends [12] a value of $r_{s}=2.8 a_{0}$. Taking $r_{s}=3 a_{0}$ together with $D_{\mathrm{op}}=1.78$ and $D_{\mathrm{s}}=78.5$ (at $300 \mathrm{~K}$ ) results in

$A=6.80 \mathrm{eV}$ and $B=4.48 \mathrm{eV}$.

In fig. 3 we present the size dependence of EVBE and EABE on $n^{-1 / 3}$. For the dependence of EABE on $n^{-1 / 3}$ we have also incorporated the experimental heat of solution of the excess electron in bulk water $\operatorname{EABE}(\infty)=\Delta H=-1.7 \mathrm{eV}[32]$, which together with eq. (13) results in the relation $\operatorname{EABE}(\mathrm{eV})=$ $-1.70+4.48 n^{-1 / 3}$. This relation is reasonably well obeyed (within 20\%) for large $n=64$ and 128 clusters (fig. 3). The QUPID data for EVBE in $n=64$ and 128 clusters give a good fit to the relationship (fig. 3) $\operatorname{EVBE}(\mathrm{eV})=-4.90+6.80 n^{-1 / 3}$, with the size dependence being well accounted for in terms of the dielectric model, eqs. (10)-(13). 


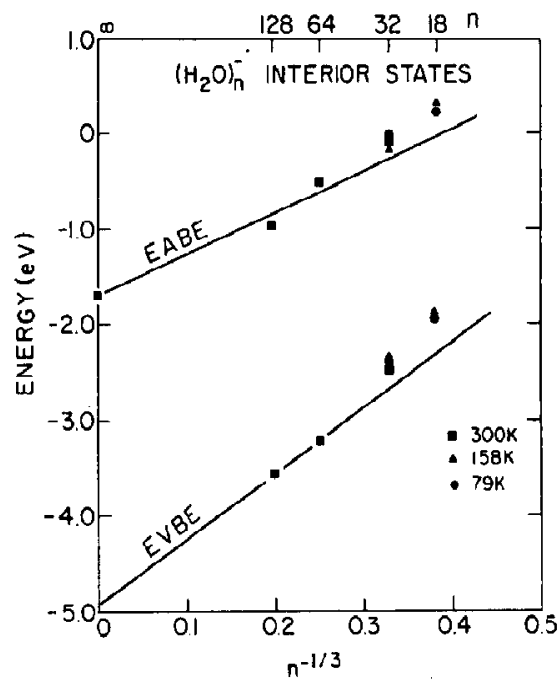

Fig. 3. The dependence of the vertical and adiabatic binding energies of interior excess states in $\left(\mathrm{H}_{2} \mathrm{O}\right)_{n}^{-}$clusters on $n^{-1 / 3}$. The upper and lower sets of points represent EABE and EVBE data, respectively, at different temperatures as indicated on the figure. The black square for EABE at $n=\infty$ corresponds to the experimental heat of solution of an electron in water [32]. The upper straight line corresponds to $\operatorname{EABE}(\mathrm{eV})=-1.70$ $+4.48 n^{-1 / 3}$ while the lower straight line corresponds to $\operatorname{EVBE}(\mathrm{eV})=-4.90+6.80 n^{-1 / 3}$.

We have provided a quantitative analysis of the size dependence of the vertical and adiabatic binding energies of an excess electron in large water clusters. Our analysis manifests the central role of the onset of long-range polarization interactions in the gradual transition from interior excess electron states in clusters to the solvated electron in bulk water. This information is of central importance in establishing the interrelationship between microscopic solvation effects in finite systems and in macroscopic polar fluids.

This research was supported by the US DOE under Grant No. FG05-86ER45234 (to UL) and by Grant No. 85-00361 of the US-Israel Binational Science Foundation, Jerusalem (to JJ and UL).

\section{References}

[1] W. Weyl, Ann. Phys. 197 (1863) 601.

[2] E.J. Hart and J.W. Boag, J. Am. Chem. Soc. 84 (1962) 4090.

[3] G. Lepoutre and M. Sienko, eds., Solutions metal-ammonia (Benjamin, New York, 1964).
[4] J.J. Lagowski and M. Sienko, eds., Metal-ammonia solutions (Butterworths, London, 1970).

[5] J. Jortner and N.R. Kestner, eds., Electrons in fluids (Springer, Berlin, 1973).

[6] Can. J. Chem. 55 (1977) 1795-2277.

[7] J. Phys. Chem. 88 (1984) 3699-3913.

[8] J. Jortner, J. Chem. Phys. 30 (1959) 839.

[9] D.A. Copeland, N.R. Kestner and J. Jortner, J. Chem. Phys. 53 (1970) 1189.

[10] J. Jortner, Ber. Bunsenges. Physik. Chem. 75 (1971) 646.

[11] M. Newton, J. Chem. Phys. 58 (1973) 5833.

[12] M. Newton, J. Phys. Chem. 79 (1975) 2795.

[13] H. Haberland, H. Langosch, H.G. Schindler and D.R. Worsnop, Surface Sci. 156 (1985) 157.

[14] H. Haberland, H.G. Schindler and D.R. Worsnop, J. Chem. Phys. 81 (1984) 3742.

[15] J.V. Coe, D.R. Worsnop and K.H. Bowen, J. Chem. Phys., to be published.

[16] M. Knapp, O. Echt, D. Kreisle and E. Recknagel, J. Chem. Phys. 85 (1986) 636.

[17] M. Knapp, O. Echt, D. Kreisle and E. Recknagel, J. Phys. Chem. 91 (1987) 2601.

[18] M. Parrinello and A. Rahman, J. Chem. Phys. 80 (1984) 860.

[19] J. Bartholomew, R. Hall and B.J. Berne, Phys. Rev. B 32 (1985) 548 .

[20] M. Sprik, M.L. Klein and D. Chandler, Phys. Rev. B 32 (1985) 545.

[21] U. Landman, D. Scharf and J. Jortner, Phys. Rev. Letters 54 (1985) 1860.

[22 ] C.D. Jonah, C. Romero and A. Rahman, Chem. Phys. Letters 123 (1986) 209.

[23] A. Wallquist, D. Thirumalai and B.J. Berne, J. Chem. Phys. 85 ( 1981$) 6404$.

[24] D.F. Coker, B.J. Berne and D. Thirumalai, J. Chem. Phys. 86 (1987) 5689.

[25] J. Schnitker and P.J. Rossky, J. Chem. Phys. 86 (1987) 3471.

[26] U. Landman, R.N. Barnett, C.L. Cleveland, D. Scharf and J. Jortner, J. Phys. Chem. 91 (1987) 4890.

[27] R.N. Barnett, U. Landman, C.L. Cleveland and J. Jortner, Phys. Rev. Letters 59 (1987) 811.

[28] R.N. Barnett, U. Landman, C.L. Cleveland and J. Jortner, J. Chem. Phys. to be published.

[29] J.R. Reimers, R.O. Watts and M.L. Klein, Chem. Phys. 64 (1982) 95.

[30] J.R. Reimers and R.D. Watts, Chem. Phys. 85 (1984) 83.

[31] B.R. Rao and N.R. Kestner, J. Chem. Phys. 80 (1984) 1587.

[32] G. Lepoutre and J. Jortner, J. Phys. Chem. 76 (1972) 682.

[33] P.R. Antoniewicz, G.T. Barnett and J.C. Thompson, J. Chem. Phys. 77 (1982) 4573.

[34] V.M. Nabutovskii and D.A. Romanov, Soviet J. Low Temp. Phys. 11 (1985) 278.

[35] P. Stampfli and K.H. Bannemann, Phys. Rev. Letters 58 (1987) 2635.

[36] J. Jortner, Mol. Phys. 5 (1962) 257. 